This item was submitted to Loughborough's Institutional Repository (https://dspace.lboro.ac.uk/) by the author and is made available under the following Creative Commons Licence conditions.

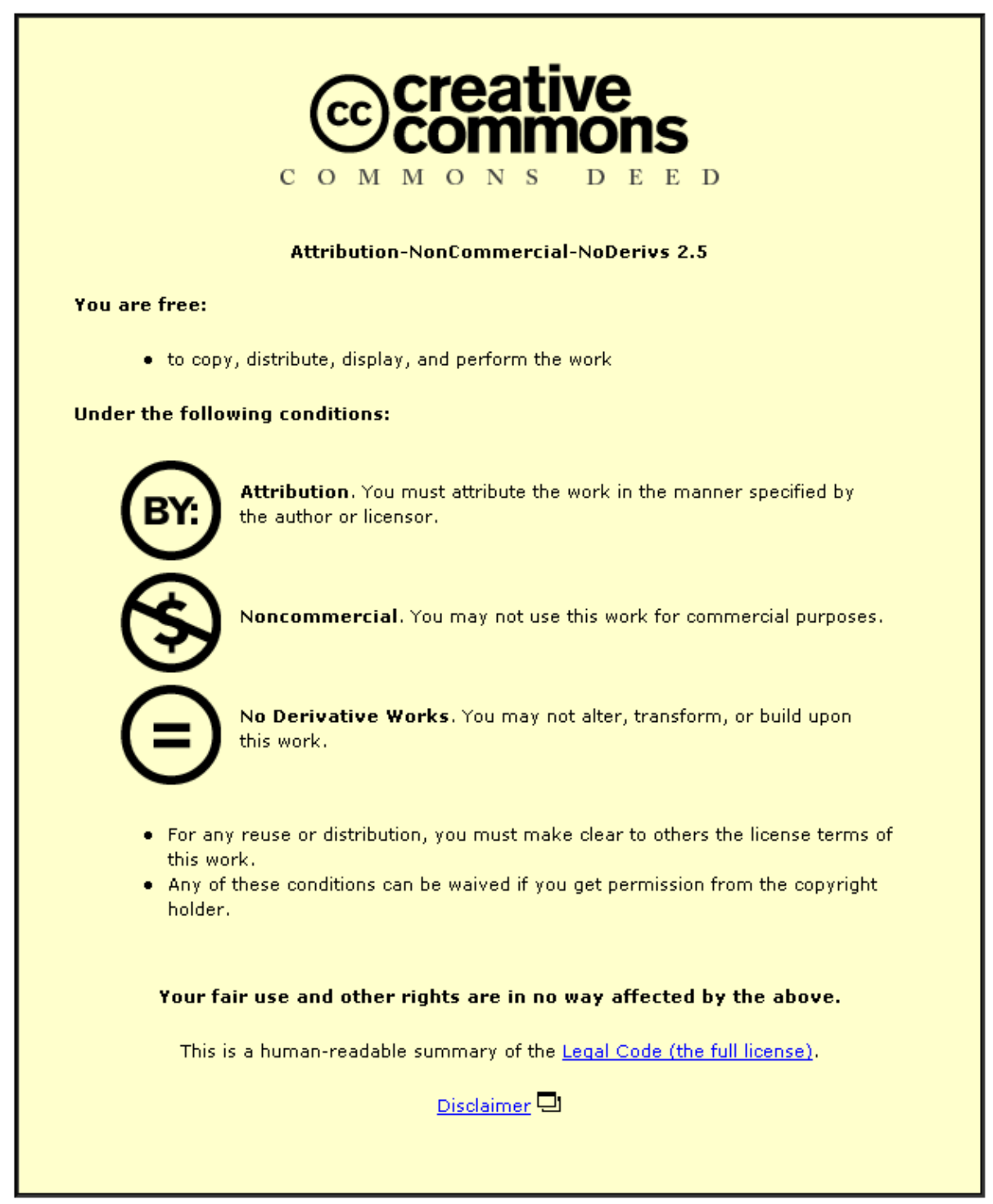

For the full text of this licence, please go to: http://creativecommons.org/licenses/by-nc-nd/2.5/ 


\title{
Electrical Conductive Characteristics of ACA Bonding: A Review of the Literature, Current Challenges and Future Prospects
}

\author{
Guangbin Dou, David C. Whalley and Changqing Liu \\ Wolfson School of Mechanical and Manufacturing Engineering, \\ Loughborough University, Leicestershire LE11 3TU, UK. \\ E-mail: gb.dou@lboro.ac.uk
}

\begin{abstract}
Anisotropic Conductive Adhesives (ACAs) have been used in fine pitch electronics packaging for over a decade and provide a high density and low temperature bonding method in a range of niche applications. The principal objective of this paper is to provide significant insights into the basic conductive characteristics of ACAs based on a review of previously reported scientific research, and to identify the current challenges and future prospects for this technology. In order to provide a concise, structured overview of this topic, many detailed conductive models, mathematical solutions and research methodologies are presented based on the reviewed literature. These models can partially explain the conductive mechanisms of an ACA particle, but make a number of important simplifying assumptions. However, one model was developed and can be used to explain the conductive mechanism of an ACA particle more successfully. In conclusion, existing computational models, mathematical models and physical models have been used to estimate the resistance of an ACA particle and the particle contact area, and therefore constriction resistance, for a given degree of particle deformation, thereby almost achieving a model for the whole resistance of an ACA joint. The paper will close by identifying other research challenges remaining for this important electronics interconnection technology.
\end{abstract}

\section{Introduction}

ACAs are conceptually a simple connection method for high density electrodes. They offers certain advantages in terms of low cure temperature, high interconnect density, and reduced package size [1]. ACAs are experiencing a significant growth in use, since they are well suited to a number of niche applications, such as in smart cards, in hybrid circuit and chip on board die-attach, and in LCD display assembly.

Electrical conduction through an ACA is achieved by the mechanical deformation of conductive particles under pressure and heat. In order to understand the electrical and mechanical behavior of ACA materials, three key issues require to be considered. First, sufficient conducting particles must be trapped during the bonding process to guarantee electrical contact with both conductor surfaces. Second, the degree of de- formation of the conducting particles during bonding determines the contact area and therefore the electrical characteristics of the interconnection. Third, the residual stress state after assembly significantly influences the mechanical performance under testing conditions and service [1].

Locking in the correct degree of deformation of the conductive particles to produce low resistance contacts is an essential factor in achieving good electrical conductive characteristics in an ACA assembly. These contacts are similar in nature to the stationary contacts present in separable electrical connectors, but in an ACA the normal force is maintained by the cured adhesive resin rather than by external mechanical means. While the complex effects of mechanical contact on the electrical resistance of the stationary contacts between the pads and the particles have been briefly addressed in a number of papers [2]-[5], no systematic understanding of these effects has been achieved. There are still significant gaps in the existing understanding of other factors governing the achievement of stable low resistance interconnections in an ACA.

\section{ACA Types and Their Typical Composition}

Anisotropic Conductive Adhesives are designed to achieve fine pitch electronic assembly, providing electrical paths as well as mechanical connections between the conductive pads on electronic components and substrates.

There are two forms of ACA, films (ACFs) and pastes (ACAPs) as shown in figure 1. These two kinds of ACAs typically have similar compositions, but are delivered to the user in a different form. Figure 1 shows the main categories of ACA material. Paste materials are applied by either printing (screen, stencil or dip techniques) or dispensed with a syringe. Film materials are supplied by the adhesive manufacturer in a reel and the end-user must have dedicated equipment to cut, align and tack the adhesive into position on the substrate [6]. Two types of adhesive matrix are available: thermoplastic and thermosetting. Thermoplastic adhesives are rigid materials at temperature below the glass transition temperature $(\mathrm{Tg})$ of the polymer, but will begin to flow at temperatures above the Tg. Thermosetting adhesives cure either through application of heat or UV radiation and the curing process 


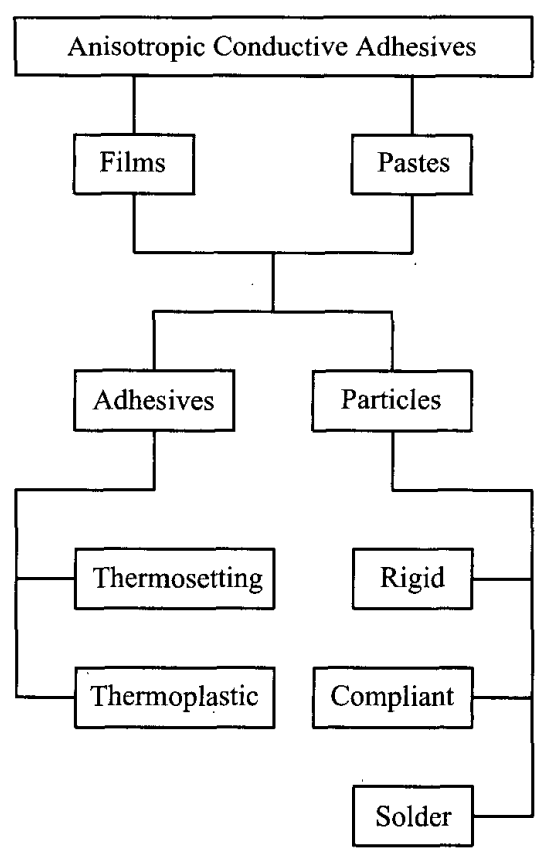

Fig. 1. ACA types and their typical compositions.

cannot be reversed. There are three types of particles used in ACAs, rigid particles, compliant particles and solder particles [6]. ACA particles are generally dispersed randomly in the polymer at a low density, however, ACFs have been developed where the particles are uniformly separated in the same non-conductive plane [7]. These two types of material are referred to as random ACAs, and ordered ACFs respectively.

\section{Conduction Mechanisms in ACA Assemblies}

Electrical conduction through ACAs is achieved by tiny metal or metal coating particles contained within the cured polymer. As described by Whalley et al [8], these materials rely upon the trapping of the conductive particles between the conductive pads on the two parts being connected, followed by the solidification of the adhesive thereby locking in residual stresses to ensure retention of sufficient contact force to create stable and low resistance electrical connections.

Figure 2 shows an ACA material consisting mainly of an adhesive layer containing a random dispersion of fine conductive particles. In general, ACA materials are prepared by dispersing electrically conductive particles in an adhesive matrix at a concentration far below the percolation threshold. The concentration of particles is controlled such that enough particles are present to provide conduction in the Z-direction while too few particles are present to achieve percolation conduction in the X-Y plane [6]. Therefore,

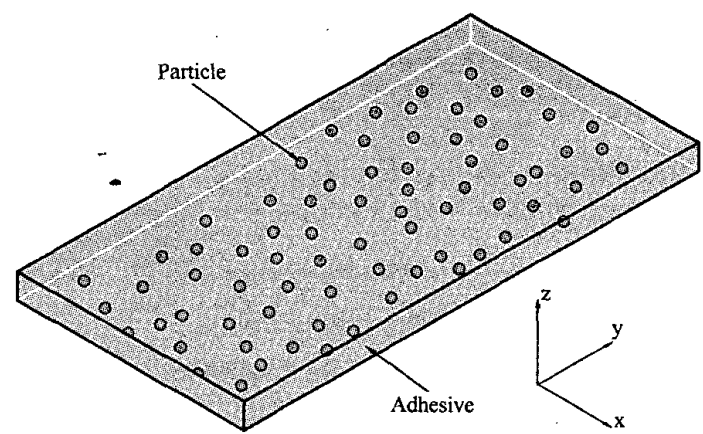

Fig. 2. Schematic of an Anisotropic Conductive Adhesive.

the conductivity of these materials is restricted to the Z-direction (perpendicular to the plane of the board) with electrical isolation provided in the X-Y plane.

A single ACA connection includes three parts: the component bump, ACA particles, and the substrate pad, as schematically shown in figure 3 . A crosssection profile of an ACA joint including one particle is shown in figure 4, which shows the real scale of a typical particle in relation to its surrounding joint parts in a typical flip-chip application.

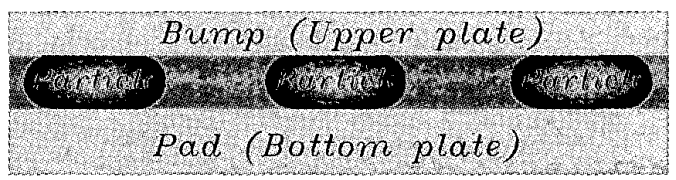

Fig. 3. Schematic Cross-section of an ACA connection.

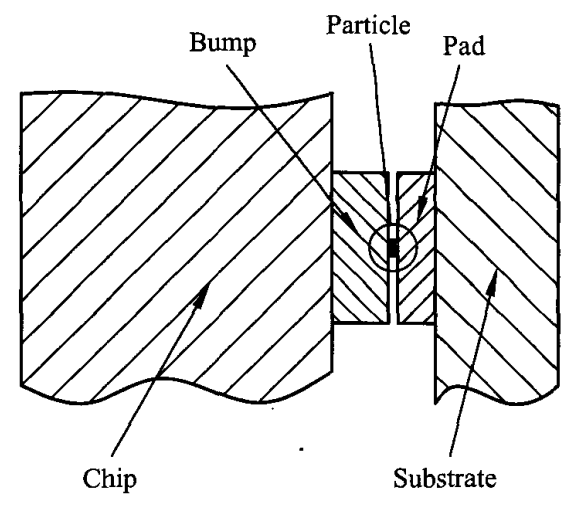

Fig. 4. A single current path in an ACA joint.

Dou et. al. treated DC current path through the bump, the single particle, and the pad as being divided into five parts, which can be represented as five independent resistors as shown in figure 5 [9]. The two $R_{\text {contact }}$ s are the stationary contact resistances 
between the particle and the pads. Stationary contact resistance is a complex problem that is affected by many factors.

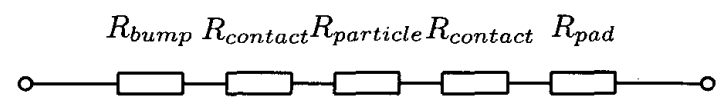

Fig. 5. The five resistances in the DC current path [3].

\section{Stationary Electric Contacts}

The conduction mechanisms for ACA assemblies are dependent on the pad materials and the type of particles that are trapped between the pads, which determine the interface properties of the electrical contact and the extent of particle deformation.

\section{A. Stationary Electric Contact Theory}

The surface topography of a typical gold coating chip bump is shown in figure 6 . Even with excellent polishing or plating, the micro-topography of such a metal surface cannot be perfectly smooth. The contact

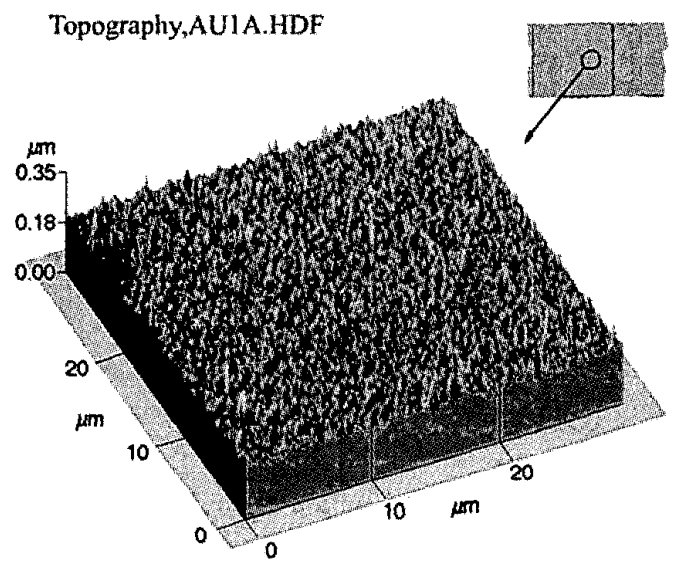

Fig. 6. The surface topography of a gold coated chip bump.

area between two metal surfaces is therefore not as large as it appears because of the asperity of the metal surface.

Holm's stationary electric contact theory [10] is widely recognized as being applicable in this area. The term electric contact means a releasable junction between two conductors, which is apt to carry electric current and a stationary contact is where two metal conductors are held in contact via an external load. The load that presses the contact members together is the mechanical load or simply the load, $P$, or normal force. If the contact members were infinitely hard, the load could not bring them to touch each other in more than three points. However since actual materials are deformable, the contact points become enlarged

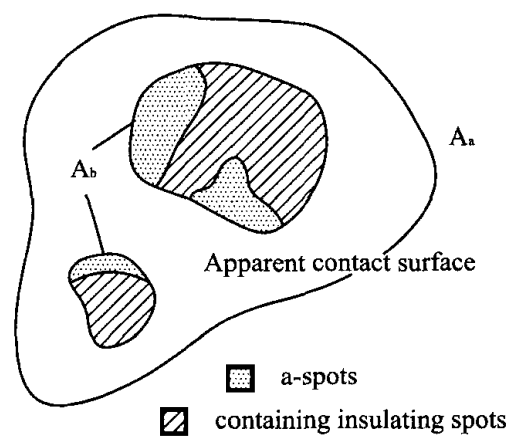

a)

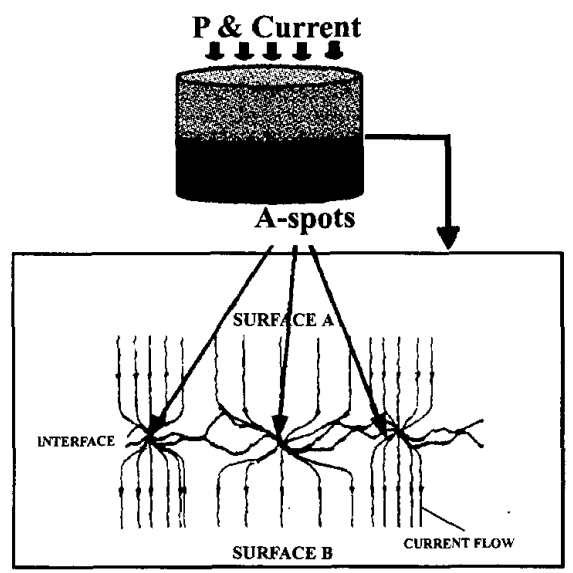

b)

Fig. 7. Apparent contact surface $A_{a}$; load-bearing contact area $A_{b}$, containing insulating spots (shaded) and conducting spots, i.e., a-spots (dotted), after [10] and [16].

into small areas and simultaneously new contact points may occur. As shown in figure 7 , the sum of all these areas and spots is the load bearing area, $A_{b}$. The whole covered area is called the apparent contact area, $A_{a}$, upon which the pressure is finite. $A_{b}$ can be generated merely by elastic deformation. But, because of the unevenness, the contact members, even though they may be nominally flat, actually touch each other in areas that are more or less plastically generated. They then satisfy the following:

$$
P=\xi H A_{b}
$$

Where usually $0.2<\xi<1$ ( $\xi$ is a factor affected by the asperity of the metal surface.) and $H$ is the contact hardness $\left(\mathrm{N} / \mathrm{mm}^{2}\right) . A_{b}$ may be of much smaller order of magnitude than the apparent contact area.

The contact resistance due to this restricted contact area is known as the constriction resistance, $R_{C}$, 
which is the consequence of the current flow being constricted through the small conducting spots. The term $a$-spot is usually used for the conducting contact areas, referring to the radius $a$ of a circuit contact area. In addition to this constriction resistance, an alien film between the particles and pads may give rise to an additional film resistance, $R_{f}$. The surface $A_{b}$ usually is partly covered by insulating tarnish films and then only a fraction of $A_{b}$ has metallic or quasimetallic contact, producing a relatively small film resistance because of the tunneling effect. Thus, the total contact resistance, $R$, is the sum of the constriction resistance $R_{c}$ resulted from the two contact members and the film resistance $R_{f}$ :

$$
R=R_{c}+R_{f}
$$

The contacts in an ACA joint are a form of stationary electric contact, except that they are retained by the cured or solidified polymer instead of external mechanical means. Some authors have suggested that the stationary electric contact resistance is the main portion of the whole ACA joint [6][11]. Liu [6] assumed that all particles touching both substrate and component surfaces make electrical contact, then $A_{b}$ is equivalent to the area of the electrically conducting spot such that:

$$
A_{b}=\pi n a^{2}
$$

where $a$ is equivalent to the area of the electrically conducting spot (i.e., the radius of the spot formed at the substrate/ particle interface) and $n$ is the number of spots. If the particle concentration is sufficiently low, then each particle contributes to $R_{c}$ independently and from Holm's theory:

$$
R_{c}=2\left(\frac{\rho}{4 n a}\right)
$$

where $\rho$ is the resistivity of the particles.

In reference [6], it is assumed $\xi=1$. By combining Equation (1), (3) and (4), it is seen that:

$$
R_{c}=0.0886 \rho(H / n P)^{\frac{1}{2}}
$$

From Equation 5, it can be seen that for a certain particle, if $\xi=1$, the contact resistance is determined by the normal force, $P$ (per particle).

\section{B. ACA Stationary Electric Contacts}

As already noted ACA assembly creates a form of stationary electric contact where a non-conductive adhesive maintains the contact force. Kristiansen [12] reported the characterization of such electrical contacts using Holm's contact theory. Several factors that may affect the ACA electric contacts were explored in his study.

The indentation in an isotropic semi-infinite body, produced by a spherical indenter is schematically

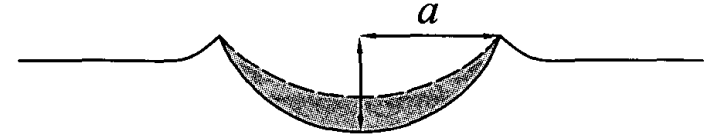

Fig. 8. The indentation in an isotropic semi-infinite body, produced by a spherical indenter, after [12].

shown in figure 8 . The heavy line represents the indentation before removal of the force on the indenter ball. The dashed line represents the final remaining deformation in the surface. The space between the two lines represents the elastic (load bearing) deformation of the indentation. The stress is most inhomogeneous at the rim, and the plastic deformation starts in this area. In this way, the "mouth" of the indentation remains visible. The mouth area $\pi a^{2}$ is practically equivalent to the load bearing area, which means Equation (1) can be written as:

$$
P=H \pi a^{2}
$$

However, in a real contact not all the contact spots will experience a stress above the yield stress of the material. The total area of the contact spots during pressure will therefore be somewhat larger than expected from the contact force. Whereas the observable area of indentation after the load has been removed will be smaller.

Kristiansen [12][13] explained the effect of contact films present in the bearing area, $A_{b}$. In an ACA connection, films of different composition may be present:

- Pre-tarnish film, which typically is a chemisorbed monolayer of oxygen on the metal surface. This can be present on any types of ACA particle.

- Tarnish, where one constituent of the film is the matrix metal, as a metal oxide. This may be found on $N i$ and $A g$ ACA particles.

- Alien films, comprised of "foreign" materials such as water, hydrocarbons, etc. A thin layer of adhesive may also be trapped in the ACA contact area.

The thickness of the tarnish film can be a monolayer, a thin protective or passivating film, which stops growing at a certain thickness. Other films can grow continuously, but diffusion processes will often inhibit their growth rate. The film resistance can be controlled by the tunneling resistance. In such cases, the current will be able to penetrate the thin insulating layers by tunneling due to the wave like properties of the electron, however, when the film in the contact area is very thin then the constriction resistance, and not the tunneling resistance, dominates the contact resistance in the quasimetallic contact area.

The chemisorption of oxygen was discussed by Kristiansen [12][13]. Chemisorption is a more complicated 
phenomenon, where oxygen is often involved. Initially the oxygen molecule may be physisorbed, but within a fraction of a second, the molecule is dissociated into atoms which become chemically bonded to the dangling bonds on the metal surface. The oxide layer will continue to grow when the bond energy between the oxygen and metal represents a larger energy than the sum of the metal-metal bond and the oxygenoxygen bond in the oxygen molecule. This results in a parabolic rate of oxidation [12].

During the curing/setting of the ACA assembly, tarnish films may be broken due to the asperity of the contact surface. When one member of the contact is deformed plastically, groups of atoms on the surface will be moved relative to each other. The result is that the tarnish layer is ruptured, creating spots of bare metal surface [12]. Figure 9 shows a model of a

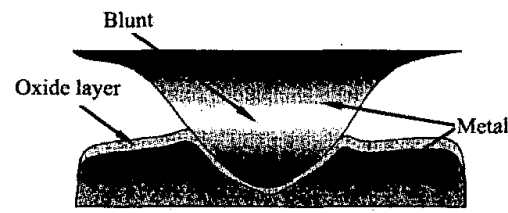

Fig. 9. A schematic model of a possible deformation of the oxide, after [12].

possible deformation of the oxide. The model shows that it is the initial pointed spike that causes the most significant thinning of the oxide, whereas a blunt elevation causes a much smaller reduction in the oxide thickness.

\section{Stationary Electric Contact Model}

Yim et al. [14] derived an analytical model based on Holm's theory and Hertz's classic elastic theory, which describes the interconnection resistance of an $\mathrm{ACF}$ in terms of sample geometry, particle size and applied pressure. In the model, the constriction resistance $R_{C R}$ has been shown to be:

$$
R_{C R}=\frac{\rho_{1}+\rho_{2}}{2 d}
$$

where $\rho_{1}, \rho_{2}$ are the intrinsic resistivity of the metal conductive particles and substrate respectively, and $d$ is the diameter of the contact spot area [10]. The magnitude of the deformation for a given pressure is determined by the number, the contact hardness and the diameter of the conductive particles. In considering these factors, the radius of the contact area between conductive particles, with number of $n$, and a flat substrate under an applied force $F$, can be obtained using elasticity theory [15]:

$$
a=1.63\left(\frac{F D}{n E^{*}}\right)^{1 / 3}
$$

Substituting Equation (8) into Equation (7), we get the constriction resistance:

$$
R_{C R}(\text { elastic })=0.347\left(\rho_{1}+\rho_{2}\right)\left(\frac{n E^{*}}{F D}\right)^{1 / 3}
$$

The behavior of the contacts in an ACA assembly was studied in a similar way by Chiang et al. [16]. Figure 7-b shows the surface contact mechanical and electrical behavior of a metal to metal stationary contact. The $A$-spots instead of the entire nominal contact surface constrict the electrical current between these two objects. Since the conductive particles in an ACA/ACF are very small (diameter of around $5 \mu \mathrm{m}$ ), one approach that has been used is to assume that each of the conductive metal particles can be treated as an $A$-spot. Therefore, after considering classic elastic theory, the constriction resistance is the same as in Equation 9, which indicates that the electrical resistance can be estimated if the material properties of the two contact bodies, the contact pressure and the radius of the deformed surface are known.

Two kinds of ACA contact conduction, singleparticle conduction and multiple-particle conduction, were studied by Shi et al. for solid particles [17].

- Single-particle conduction

If a single particle is placed between two conductors as shown in figure 3 , the total resistance of the system can be written as (see figure 5):

$R=R_{\text {bump }}+R_{\text {contact }}+R_{\text {particle }}+R_{\text {contact }}+R_{\text {pad }}$

Shi et. al. extended this to:

$R=R_{p u}+\left(R_{c u}+R_{t u}\right)+R_{b l k}+\left(R_{t b}+R_{c b}\right)+R_{p b}$

where $R_{p u}$ and $R_{p b}$ are the two constriction resistances of the plates, $R_{c u}$ and $R_{c b}$ are the constriction resistances between the plates and the particle, $R_{t u}$ and $R_{t b}$ are the tunneling resistances, and $R_{b l k}$ is the bulk resistance of the particle.

Both the upper and lower conductors can be considered to be circular, with finite diameters of $b_{u}$ and $b_{b}$, respectively. The equipotential surfaces around each plate then are hemispherical, with the center being the center of the plate. The current flows radially, normal to the equipotential surfaces as schematically shown in figure 10. The constriction resistance of $R_{p u}$ is then readily given by:

$$
R_{p u}=\int_{b u}^{\infty} \frac{\rho d \xi}{2 \pi \xi^{2}}=\frac{\rho}{2 \pi b_{u}}
$$

and similarly:

$$
R_{p b}=\frac{\rho}{2 \pi b_{b}}
$$




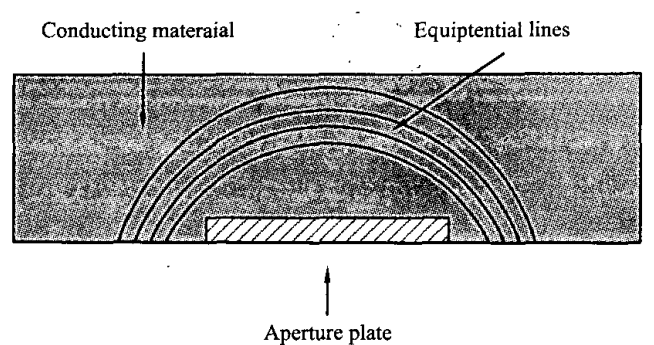

Fig. 10. Schematic for the calculation of the constriction resistance of a conducting plate of finite size [17].

In a similar way, the constriction resistance of the contact is given by:

$$
R_{c u}=\int_{r u}^{b_{u}} \frac{\rho d \xi}{2 \pi \xi^{2}}=\frac{\rho}{2 \pi}\left(\frac{1}{r_{u}}-\frac{1}{b_{u}}\right)
$$

where $r_{u}$ is the radius of the contact between the particle and the upper plate. Similarly, the constriction resistance of the contact between the particle and the lower plate is:

$$
R_{c b}=\frac{\rho}{2 \pi}\left(\frac{1}{r_{b}}-\frac{1}{b_{b}}\right)
$$

where $r_{b}$ is the contact radius.

Using figure 11 as a reference, one can obtain the bulk resistance of the particle $R_{b l k}$ :

$$
R_{b l k}=\int_{-\sqrt{r^{2}-r_{b}^{2}}}^{\sqrt{r^{2}-r_{b}^{2}}} \frac{\rho d x}{\pi\left(r^{2}-x^{2}\right)}=\frac{\rho K\left(r, r_{u}, r_{b}\right)}{2 \pi r}
$$

where:

$K\left(r, r_{u}, r_{b}\right)=\ln \left[\frac{\left(r+\sqrt{r^{2}-r_{u}^{2}}\right)\left(r+\sqrt{r^{2}-r_{b}^{2}}\right)}{\left(r-\sqrt{r^{2}-r_{u}^{2}}\right)\left(r-\sqrt{r^{2}-r_{b}^{2}}\right)}\right]$

By substituting Equations (12)-(17) into Equation (11), the total resistance of a single-particle system can be written as:

$$
R=\frac{\rho}{2 \pi r_{u}}+\frac{\rho}{2 \pi r_{b}}+R_{t u}+R_{t b}+\frac{\rho K\left(r, r_{u}, r_{b}\right)}{2 \pi r}
$$

- Multiple-particle conduction

When multiple particles are placed between the conducting plates, as schematically illustrated in figure 12 , the total resistance for all the particles placed between the plates $R_{2}$ can be obtained by using the fact that the particles are arranged in parallel. Hence $1 / R_{2}=\sum 1 / R_{1} ;$ where $R_{1}$ is a sum of the constriction resistance and the tunneling resistance of an isolated particle. On the other hand, the resistance of the entire system, $R$, is given by $R=R_{2}+R_{p u}+R_{p b}$, since the particles and the plates are arranged in series.

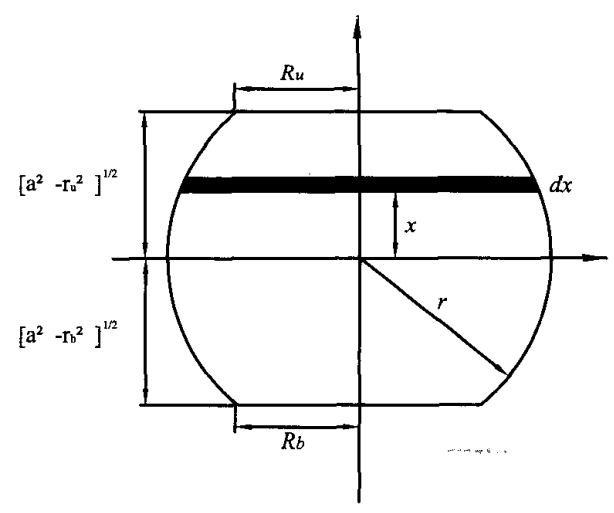

Fig. 11. Schematic for the calculation of the bulk resistance of a particle [17].
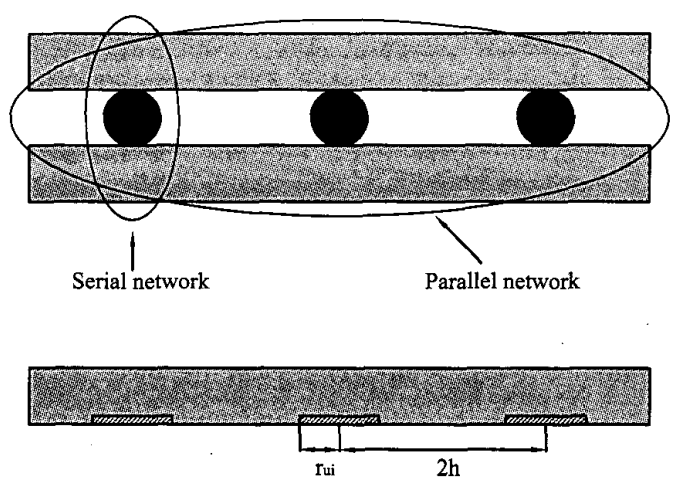

Fig. 12. Schematic of electrical conduction when multiple particles are placed between the plates and the associated contacts between the particles and the plates [17].

In a similar way to that for single particle conduction, Shi et al. [17] calculated the total resistance of the entire system. It can be expressed as:

$$
R=\left[\sum_{i}^{N} \frac{1}{R_{1, i}}\right]^{-1}+\frac{\rho}{2 \pi b_{u}}+\frac{\rho}{2 \pi b_{b}}
$$

To sum up, the electric contact models reviewed here are proposed to explain the resistance of the stationary contacts in ACA joints, and they are all based on the assumption that the $a-s p o t$ is equal to the whole apparent contact area, which is different Holm's theory, where the total $a-$ spot area is much smaller than the apparent contact area. Such discussion can be found in reference [18]. There is insufficient experimental data in the literature to conclusively demonstrate whether this approach is adequate, but there is some evidence to suggest that in practice the resistances are higher [11][18]. 


\section{Conductive Mechanism of ACA Particles}

\section{A. ACA Particle Deformation Analysis}

Two kinds of ACA particle system, rigid particle systems and deformable (compliant) particle systems, were studied by Liu [6]. The schematics of contact mechanisms of a single particle system are shown in figure 13(a) (rigid particle) and figure 13(b) (deformable particle).
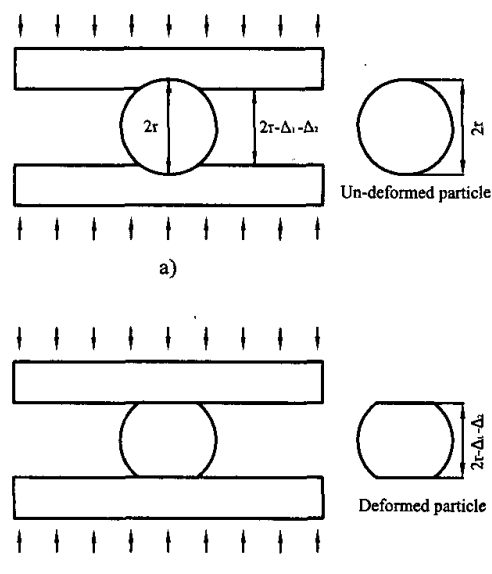

b)

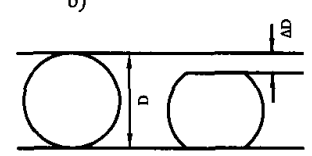

Fig. 13. (a) Schematics of a rigid particle system; (b) Schematics of a deformable particle system, after [6].

In figure 13(a), it is assumed that the particle indentation does not change the resistance of the pad metallization. Given the indentation depths of $\Delta_{1}$ and $\Delta_{2}$ in the upper and lower metal pads, respectively, the resistance of the interconnect with the particle radius $r$ is:

$$
R=\int_{-\left(r-\Delta_{1}\right)}^{r-\Delta_{2}} \rho \frac{d l}{A}=\frac{\rho}{2 \pi r} \ln \left[\left(\frac{2}{\varepsilon_{1}}-1\right)\left(\frac{2}{\varepsilon_{2}}-1\right)\right]
$$

where $\rho$ is the particle resistivity, $\varepsilon_{1}=\Delta_{1} / r$ and $\varepsilon_{2}=\Delta_{2} / r . \varepsilon_{1}$ and $\varepsilon_{2}$ measure the magnitude of particle deformation, and can be interpreted as an overall particle strain. For small strains, where $\varepsilon_{1} \ll 1$ and $\varepsilon_{2} \ll 1$, we have:

$$
R=\frac{\rho}{\pi r} \ln \left(\frac{4}{\varepsilon_{1} \varepsilon_{2}}\right)
$$

For the case where lower and upper pads have the same amount of deformation (symmetrical about horizontal plane), $\varepsilon_{1}=\varepsilon_{2}=\varepsilon$, and therefore:

$$
R=\frac{\rho}{\pi r} \ln \left(\frac{2}{\varepsilon}-1\right)
$$

And, for the symmetrical and small deformation case:

$$
R=\frac{\rho}{\pi r} \ln \left(\frac{2}{\varepsilon}\right)
$$

In the case of deformable particles figure 13(b), Equations (20) to (23) will still be valid if $\Delta_{1}$ and $\Delta_{2}$ now represent the particle deformation in the lower and upper interfaces, respectively. It should be noted that the cross-sectional area of the particles is enlarged due to the Poisson's ratio effect in the compressed conducting particles. The extent of this area enlargement depends on the cross-sectional location (or $x$-coordinate). For the purpose of bulk resistance calculations, it is assumed that area enlargement follows an average Poisson's law. The deformed area at the location $x$ becomes:

$$
A=\pi\left(r^{2}-l^{2}\right)\left[1+\frac{\nu\left(\varepsilon_{1}+\varepsilon_{2}\right)}{2}\right]^{2}
$$

where $\nu$ is the Poisson's ratio of the particle. Accordingly, Equation (20) is modified to account for the effect of Poisson's ratio on the resistance, giving:

$$
R=\frac{\rho}{2 \pi r\left[1+\frac{\nu\left(\varepsilon_{1}+\varepsilon_{2}\right)}{2}\right]^{2}} \ln \left[\left(\frac{2}{\varepsilon_{1}}-1\right)\left(\frac{2}{\varepsilon_{2}}-1\right)\right]
$$

For the symmetrical case, where lower and upper particles are compressed by the same amount:

$$
R=\frac{\rho}{\pi r(1+\nu \varepsilon)^{2}} \ln \left(\frac{2}{\varepsilon}-1\right)
$$

Equations (20) and (25) depict the closed-form resistance-deformation relationships for the rigid and the deformable particle systems. Under a deformationcontrolled scheme, these equations can be used to estimate how much deformation is required to attain a desirable level of contact resistance.

\section{B. Conduction Models of Metal Coating Polymer Par- ticles}

Määttänen [19] calculated the particle resistance as a function of particle deformation degree using a conduction model.

Starting from Ohm's law, the resistance of a conductor, $R_{P}$, is given by:

$$
R_{P}=\rho \frac{l}{A}
$$

where, $\rho$ is the resistivity of the metal coating, $l$ is the length of the conductor and $A$ is the cross sectional area of the conductor.

The degree of deformation, Deformpercent, is described by the formula:

$$
\text { Deform per cent }=100\left[1-\left(\frac{h}{2 r}\right)\right]
$$


where $h$ is the distance between the contact surfaces and $r$ is the undeformed radius of the metal coating polymer particle.

If it is assumed that the area of the metal coating remains constant during the deformation, the following results can be derived from the geometry (figure 14). The area of the spherical cap forms the metallic

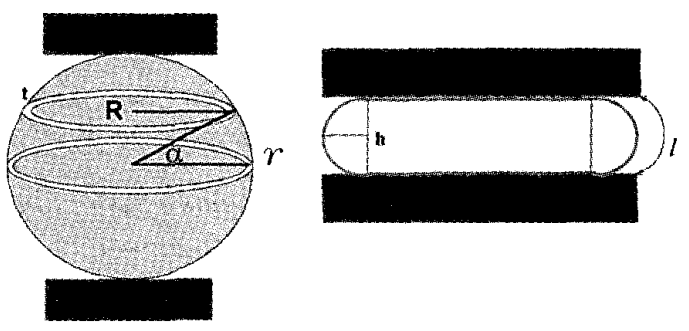

Fig. 14. Deformation model of the particle [19].

contact area of the particle and, therefore, the metallic contact area, $\mathrm{A}$, is given by:

$$
A=2 \pi R^{2}\left(1-\frac{h}{2 r}\right)
$$

And the length of the conductor, $l$, is given by:

$$
l=\frac{\pi h}{2}
$$

The average cross sectional area of the current is the average area of the thin metal "rings" around the polymer particle (figure 14). Because the resistance is inversely proportional to the cross sectional area of the conductor, the average area can be calculated as follows:

$$
\begin{array}{r}
\left(\frac{\tilde{l}}{A}\right)=\frac{\int_{0}^{\frac{\pi h}{4}} \frac{d S}{2 \pi t r \cos \alpha}}{\int_{0}^{\frac{\pi h}{4}} d s} \\
=\frac{2}{\pi^{2} h t} \ln \tan \left[\frac{\pi}{4}\left(1+\frac{h}{2 r}\right)\right]
\end{array}
$$

where $R=r \cos (\alpha)$ (figure 14), $S=r \alpha, d S=r d \alpha$ and $t$ is the thickness of the metal layer on the particle.

Since there is spherical symmetry an approximation can be made and the problem is solved using Ohm's law and the formula for the resistance of the deformed particle, $R_{p}$, is written as follows:

$$
R_{P}=\rho \frac{\pi h}{2}\left(\frac{\tilde{l}}{A}\right)=\frac{\rho}{\pi t} \ln \tan \left[\frac{\pi}{4}\left(1+\frac{h}{2 r}\right)\right]
$$

An important observation that can be made from this is that the resistance is independent of the particle size, but depends on the resistivity of the particle $(\rho)$, the thickness of metal layer $(t)$ and the degree of deformation $(h / 2 r)$.
For the solid metal particle the following approximate formula can be used [20]:

$$
R_{P}=\frac{\rho}{\pi r} \ln \left(\frac{2}{\varepsilon}-1\right)=\frac{\rho}{\pi r} \ln \left(\frac{1+\frac{h}{2 r}}{1-\frac{h}{2 r}}\right)
$$

where $\varepsilon$ is the average degree of deformation.

It can be seen from Equation (33) that for a solid particle the resistance increases when the particle size becomes smaller. The pressure does not affect the degree of deformation of hard particles, for example nickel, as much as for softer particles like gold or silver. In figure 15 the main results obtained from formulas (32) and (33) are presented, where the particle size is $10 \mu \mathrm{m}$. Figure 15 shows the large difference in parti-

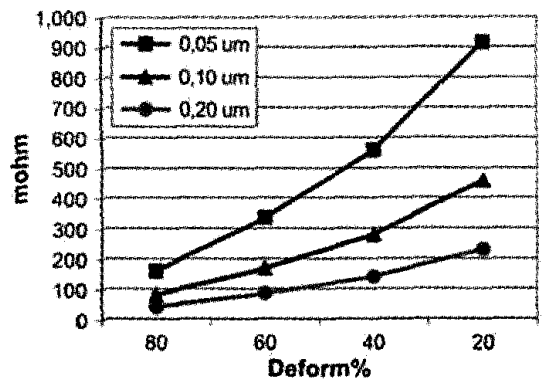

(a)

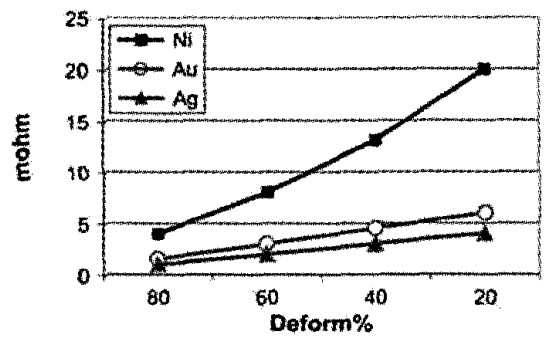

(b)

Fig. 15. Predicted particle resistance for different degree of deformation (a) for $\mathrm{Au} / \mathrm{Ni}$-polymer with various metal thicknesses, (b) for solid metal particles [19].

cle resistance between the solid metal and the $A u / N i$ coating particle. In conclusion, the thickness of the metal coating has a direct influence on the resistance. This means that there is a large difference, depending on particle type, in the number of particles estimated to be needed for a good contact. Solid gold and silver particles give a much smaller resistance compared to the nickel coating polymer particle.

Another model for the electrical conduction characteristics of metal coating ACA particles, was derived by Dou et al. [9]. According to the numerical solutions, the greater the level of particle transformation, 
the thicker the metal coating layer and the greater the resin diameter, the lower the particle resistance is. It was shown that the ACA particle resistance is !determined by the particle transformation and the particle geometries, however it is more sensitive to the transformation and the nickel layer thickness than the resin diameter and the gold layer thickness.

Here the deformation degree of an ACA particle, $K$, is defined differently to Equation (28), as shown in figure 13:

$$
k=\frac{\Delta D}{D} \times 100 \%
$$

An ACA particle, consisting of a nickel layer, a gold layer and a resin core, is shown schematically in figure 16. The particle resistance can be considered as being

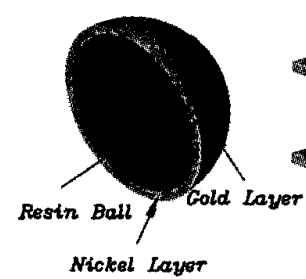

a)

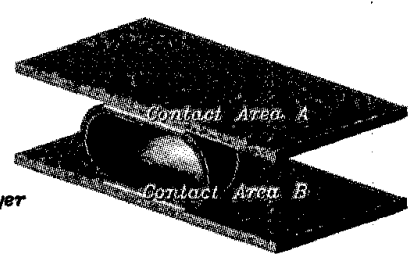

b)
Fig. 16. An ACA particle and its connection [9].

the resistance of the $N i$ layer in parallel with the $A u$ layer:

$$
\frac{1}{R_{\text {particle }}}=\frac{1}{R_{\text {nickel }}}+\frac{1}{R_{\text {gold }}}
$$

The resistance of a single ACA particle is a multiparameter function, i.e. it is a function of resin sphere diameter $d$, transformation $k$, nickel layer thickness $t_{1}$ and gold layer thickness $t_{2}$. During the transformation process, the particle volume is not changed, hence the original resin sphere volume $V_{\text {sphere }}$ is equal to the deformed resin solid volume $V_{\text {deformed }}$ :

$$
V_{\text {deformed }}=V_{\text {sphere }}=\frac{4}{3} \pi\left(\frac{d}{2}\right)^{3}
$$

Figure 17- $a$ shows a deformed resin ball, whose crosssectional profile in the $y z$-plane is illustrated in figure 17- $b$. As the illustration shows, the cross-sectional a profile consists of a rectangular area and two half circle areas. The length of the arc enclosing the right half circle area is given by:

$$
[y-R]^{2}+z^{2}=\left[\frac{h}{2}\right]^{2}, \quad(y \geq R)
$$

where $R$ is the radius $R(k, d)$ function of the top circle area, and $h$ is the height function $h(k, d)$ of the deformed resin as dimensioned in figure $17-b . h$ is defined as

$$
h(k, d)=(1-k) d
$$

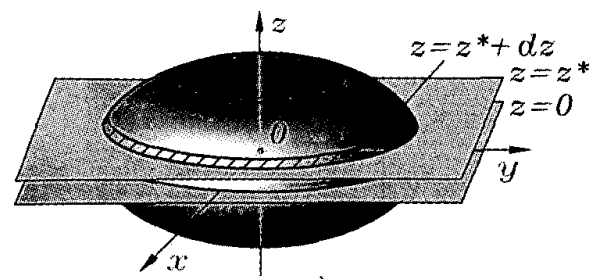

a)

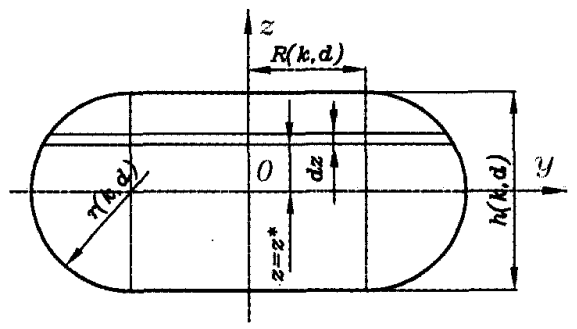

b)

Fig. 17. Deformed resin of an ACA particle [9].

As figure 17- $a$ and $b$ show, the whole deformed resin volume can be expressed with an integral equation

$$
\begin{array}{r}
V_{\text {deformed }}=\int_{-\frac{h}{2}}^{+\frac{h}{2}} V^{*} \\
=\pi \int_{-\frac{h}{2}}^{+\frac{h}{2}}\left[\sqrt{\left(\frac{h}{2}\right)^{2}-\left(z^{*}\right)^{2}}+R\right]^{2} d z
\end{array}
$$

Applying Equation (39) and (38), an equation for $R(k, d)$ is obtained:

$$
\begin{array}{r}
R(k, d)=\frac{-3 \pi(1-k)^{2}}{24(1-k)} \\
+\frac{\sqrt{\left[3 \pi(1-k)^{2} d^{2}\right]^{2}-48(1-k)\left[2(1-k)^{3} d^{2}-2 d^{2}\right]}}{24(1-k)}
\end{array}
$$

For the particle, resin coated with nickel layer and gold layer, the nickel layer is schematically shown in figure 18. In the $z$ direction, the increment in electrical resistance $d R^{*}$ over a distance $d z$ is given by:

$$
d R^{*}=\frac{d z}{\sigma S^{*}}
$$

where

$$
\begin{array}{ll}
\sigma & \text { nickel conductivity, }(m \Omega)^{-1} \\
S^{*} & \text { ringlike region area or circle area, } \mu m^{2} ; \\
d z & \text { the height of the integral unit, } \mu \mathrm{m} .
\end{array}
$$

For $-\left(\frac{h}{2}+t_{1}\right) \leq z \leq+\left(\frac{h}{2}+t_{1}\right)$, the resistance of the $\mathrm{Ni}$ layer is:

$$
R_{n i c k e l}\left(k, d, t_{1}\right)=R_{n i c k e l}^{1}+R_{n i c k e l}^{2}=\frac{2}{\sigma \pi} \int_{0}^{+\frac{h}{2}}
$$




$$
\begin{array}{r}
\frac{d z}{\left[\sqrt{\left(\frac{h}{2}+t_{1}\right)^{2}-(z)^{2}}+R\right]^{2}-\left[\sqrt{\left(\frac{h}{2}\right)^{2}-(z)^{2}}+R\right]^{2}} \\
+\frac{2}{\sigma \pi} \int_{+\frac{h}{2}}^{+\left(\frac{h}{2}+t_{1}\right)} \frac{d z}{\left[\sqrt{\left(\frac{h}{2}+t_{1}\right)^{2}-z^{2}}+R\right]^{2}}
\end{array}
$$

Similarly, the $A u$ layer resistance can be obtained:

$$
\begin{gathered}
R_{\text {gold }}\left(k, d, t_{1}, t_{2}\right)=\frac{2}{\sigma \pi} \int_{0}^{+\left(\frac{h}{2}+t_{1}\right)} \\
\frac{d z}{\left[\sqrt{\left(\frac{h}{2}+t_{1}+t_{2}\right)^{2}-(z)^{2}}+R\right]^{2}-\left[\sqrt{\left(\frac{h}{2}+t_{1}\right)^{2}-(z)^{2}}+R\right]^{2}} \\
+\frac{2}{\sigma \pi} \int_{+\left(\frac{h}{2}+t_{1}\right)}^{+\left(\frac{h}{2}+t_{1}+t_{2}\right)} \frac{d z}{\left[\sqrt{\left(\frac{h}{2}+t_{1}+t_{2}\right)^{2}-z^{2}}+R\right]^{2}}
\end{gathered}
$$

Substituting Equation (42) and Equation (43) into Equation (35), we obtain the total particle resistance:

$$
\frac{R_{\text {particle }}\left(k, d, t_{1}, t_{2}\right)=}{\frac{1}{\left[R_{\text {nickel }}\left(k, d, t_{1}\right)\right]^{-1}+\left[R_{\text {gold }}\left(k, d, t_{1}, t_{2}\right)\right]^{-1}}}
$$

where

$$
\begin{array}{ll}
k & \text { transformation factor; } \\
d & \text { resin diameter, } \mu m ; \\
t_{1} & \text { nickel layer thickness, } \mu m ; \\
t_{2} & \text { gold layer thickness, } \mu m .
\end{array}
$$
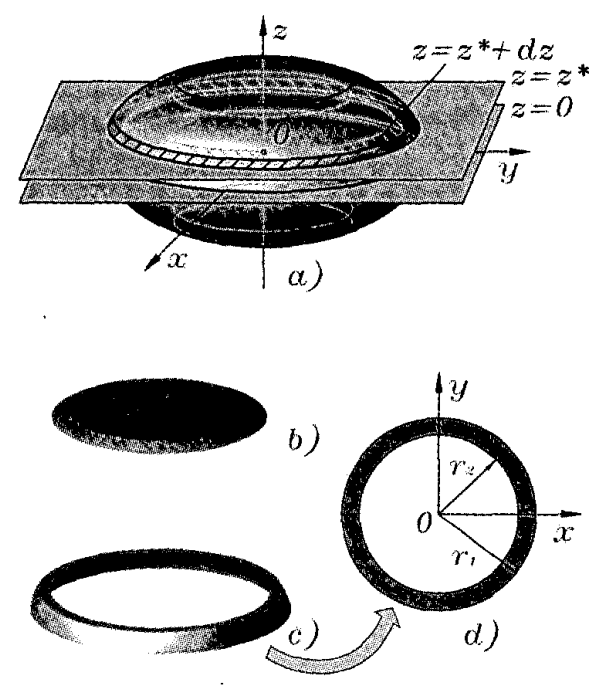

Fig. 18. Deformed nickel layer of an ACA particle [9].

- Application of the electrical resistance function.
For a type of commercial ACA, where equation (44) is a function $R_{\text {particle }}(k, 3.5,0.15,0.05)$ with one parameter, $k$, which has been calculated as shown in figure 19 for $k$ between 0.1 and 0.9 . In this case, as the function curve illustrates in the figure, the particle resistance decreases as the transformation $k$ increases. For typical commercial ACA particles, the metal lay-

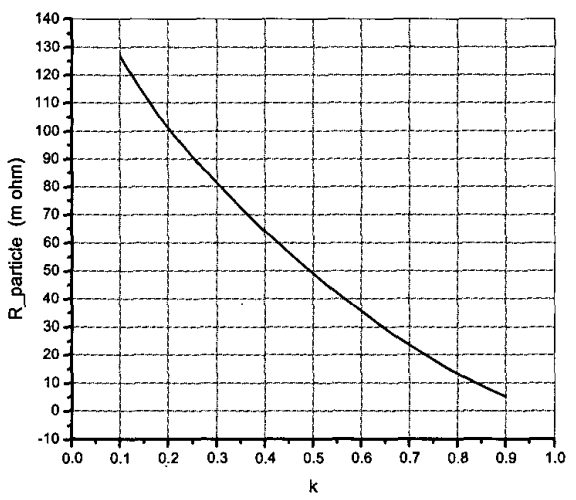

Fig. 19. A commercial particle resistance function of $k$ [9].

ers form two parallel resistors in the circuit as shown in Equation (35). However, their contributions to the particle resistance are substantially different as shown in figure 20. It can be seen from the figure that, for the

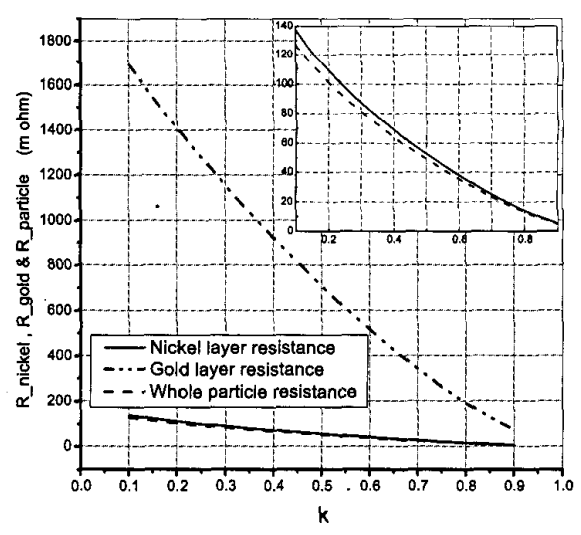

Fig. 20. Different layer resistance functions of the particle [9].

same ACA particle, the gold layer resistance is much higher than the nickel layer resistance for the same transformation degree. However, the whole particle resistance, represented by the dash curve is lower than each metal layer resistance because they are parallel resistors in the circuit. Moreover, the whole particle resistance is very close to the nickel layer resistance as shown in the figure. The gold layer therefore has little 
affect on whole particle resistance.

- Effect of Particle Size on General ACA Particle Electrical resistances

For different particles, the resin diameter represents the particle size if the metal layer thicknesses are constant. Figures 21- $a$ and $b$ show results from numerical solution of Equation (44) when $d$ is between $2 \mu \mathrm{m}$ and $10 \mu \mathrm{m}$ and $k$ is between 0.1 and 0.9 . Figure 21- $a$ is a

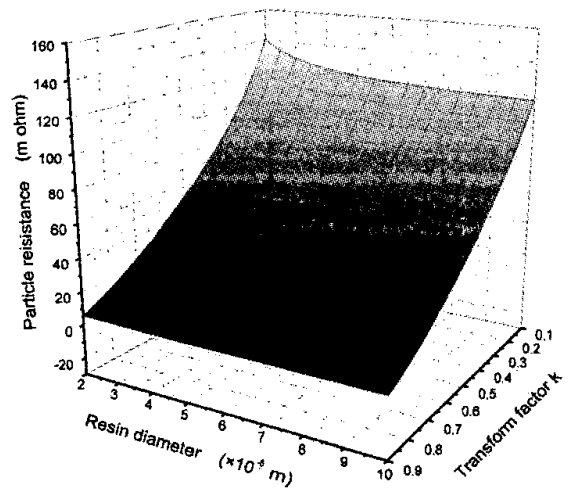

a.)

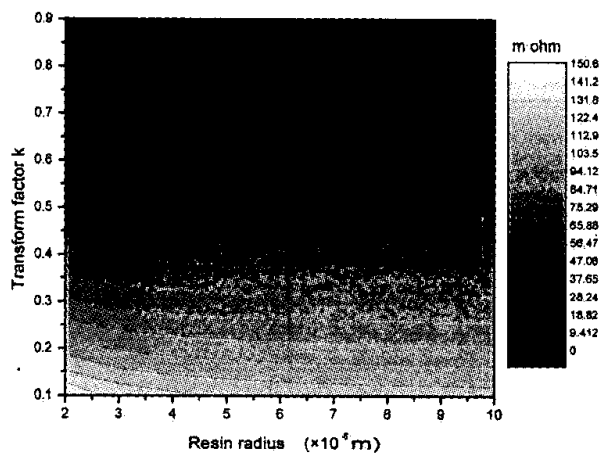

b)

Fig. 21. Particle resistance function of $k$ and $d$ [9]

3D gray scale map surface of the function, where the higher the surface is, the larger the particle resistance is. Figure 21- $b$ is a contour map of figure 21- $a$, where there are large particle resistances in the light gray areas and low resistances in the dark gray areas. It can be seen from the figures that the larger the resin particle and the deeper the transformation are, the lower the particle resistance is. However the lower resistances in the dark gray areas are not suitable to be used because they need large transformation or large resin diameter. Too large a degree of transformation will cause the particles to be crushed and too large a resin diameter will reduce the number of the trapped particles per pad, cause yield and reliability problems. In the light gray areas, the particle resistances are too large to be used. For details, see reference [9].
- Effect of Nickel Layer Thickness on General ACA Particle Electrical Resistances

The nickel layer is the main conductive function layer of an ACA particle. So, its thickness is an important factor to the particle resistance. If the resin diameter is $3.5 \mu \mathrm{m}$ and the gold layer thickness is 0.05 $\mu m$, from Equation (44) we obtain particle resistance function $R_{\text {particle }}\left(k, 3.5, t_{1}, 0.05\right)$ shown as figure 22 . From figure $22-b$, we can see when $t_{1}$ is more than

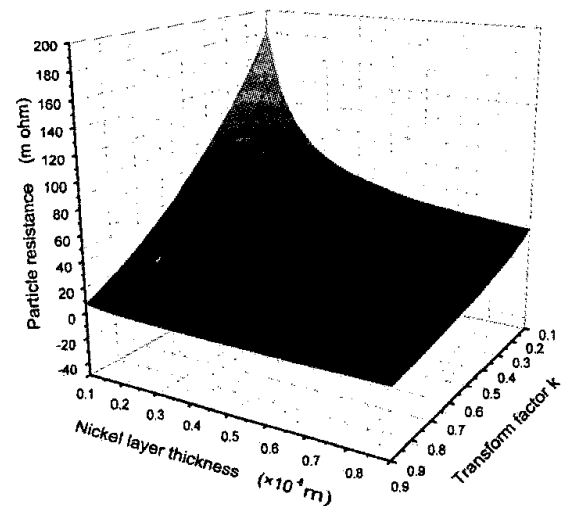

a.)

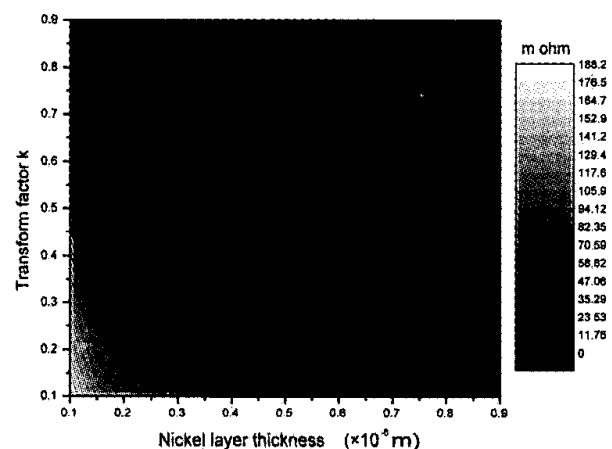

b)

Fig. 22. Particle resistance function of $k$ and $t_{1}$ [9]

about $0.15 \mu \mathrm{m}$, the resistance areas will locate in the good areas, gray areas. However it is difficulty to coat thicker layer on the resin ball and the thicker layer is not economic. The thicknesses of the nickel layers of ACA particles are usually between about $0.15 \mu \mathrm{m}$ and $0.4 \mu \mathrm{m}$. The particle resistance is sensitive to the thickness of the nickel layer as shown in figure 22- $a$, where the resistance decreases rapidly as the nickel layer thickness increases. The thicknesses of the nickel layer are normally not uniform in one particle and also in different particles, therefore the particles resistances are not the same because of the sensitive characteristics of the nickel layer resistance and the uneven metal coating layers. Moreover, the nickel layer thickness of an $\mathrm{ACA}$ particle is the main geometry that affects the 
particle resistance.

- Effect of Gold Layer Thickness on General ACA Particle Electrical Resistances

If the resin diameter is $3.5 \mu \mathrm{m}$ and the nickel layer thickness is $0.15 \mu \mathrm{m}$, from Equation (44) we obtain particle resistance function $R_{\text {particle }}\left(k, 3.5,0.15, t_{2}\right)$ as shown in figure 23- $a$ and $b$. From figure 23 and figure

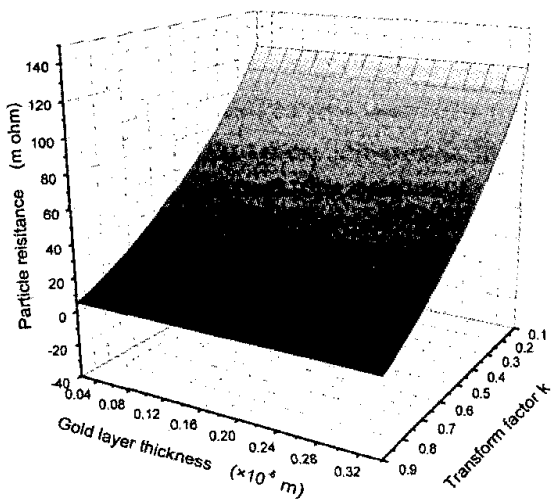

a)

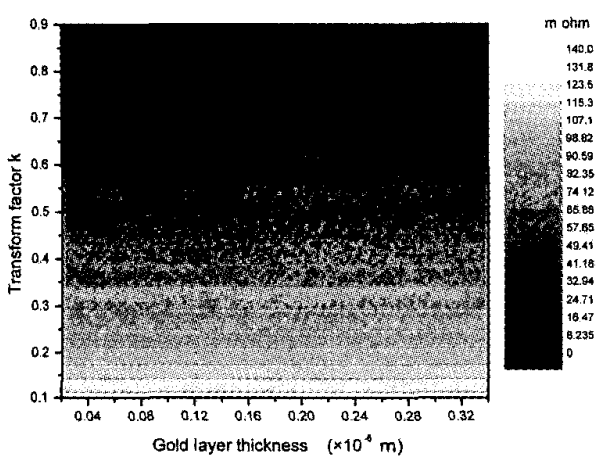

b)

Fig. 23. Particle resistance function of $k$ and $t_{2}$ [9]

19 , it can be seen that the gold layer coating of ACA the particle is not designed to improve the particle resistance, but is designed to provide good oxidation resistance, which prevents the contact area and the nickel layer from oxidizing during service helping to maintain a low contact resistance. Another advantage of the gold layer is that it is soft, which helps to increase the $a$-spot area during the deformation process and consequently reduce the contact resistance.

\section{Discussion}

The ACA contact models discussed in this paper are theoretically similar, since they are all based on Holm's contact theory and use the same assumption that the $a-s p o t$ is the whole contact area. However, the $a-$ spot should be smaller than the apparent contact area, therefore, the actual contact resistance should have been much higher than the resistance calculated from the models.

From the metal coated particle models, the particle resistance for different degrees of deformation can predict a reasonable result assuming that any cracks in the particle are vertical i.e. perpendicular to the adhesive plane, as has been observed in some experiments [9][19]. The models presented can forecast how the particle geometries, metal layers and resin ball size, affect the particle resistance for different degrees of deformation and can help to establish the optimum material properties for ACA manufacturing.

\section{Current Challenges and Future Prospects}

Many supporting experiments have been performed in order to measure the resistances of ACA joints, but these experiments are generally not able to measure individual particle resistances to support the models reviewed in this paper. The measurement of the resistance of a single ACA particle for different degrees has been proposed by the author of this paper, but undertaking such an experiment for individual particles is an important challenge. Furthermore, accurate measurement of the resistance during the particle deformation process is hard to realize.

The co-planarity effects on an ACA assembly due to the component planarity and packaging accuracy are another important issue that requires further study. This challenge was introduced by Liu [21].

As far as electrical conduction is concerned, the complex behavior of stationary electric contacts is very different to metallurgical bonds, such as solder joints, especially at such small scales, and is one of the main drawbacks to the use of ACA bonding. Therefore, the examination of the stationary electric contacts present in ACA joints is important for the conductive characteristic investigation. In other word, the conduction mechanisms for the stationary contacts of an ACA joint still need more thorough exploration.

\section{References}

1. Dudek R., Meinel S. and Schubert A., "Flow Charaterization and Thermo-Mechanical Response of Anisotropic Conductive Films," IEEE Transactions on Components and Packaging Technology, Vol. 22, No. 2, June 1999, pp177-185.

2. Williams D. J., Whalley D. C., Boyle O. A. and Ogunjimi A. O., "Anisortopic Conducting Adhesive for Electric Interconnection," Soldering \& Surface Mount Technology, No. 14, June 1993, pp4-8.

3. Yeung N. H., Chan Y. C. and Chan C. W., "Electrical Conductive Characteristics of Anisotropic Conductive Adhesiv Paritcles," Journal of Electronic.Packaging, ASME, Vol. 125, December 2003, pp624-629. 
4. Fu Y., Wang Y., Liu J., Lai Z., Chen G. and Willander M., "Experimental and Theroratical Charaterization of Electrical Contact in Anisotropically Conductive Adhesive," IEEE on Advanced Packaging, Vol. 23, No. 1, February 2000, pp15-21.

5. Fu Y, Willander M. and Liu J., "Stastics of Electric Conductance Through Anisotropically Conductive Adhesive," June 2001, pp250-255.

6. Liu J., "Conductive Adhesive for Electronics Packaging," Electrochamical Publications Ltd,' 1999, London, UK.

7. Holloway M. J., Ward M. B. and Scully L. V., "Novel Anisotropically Conductive Film for Area Array Packaging," Advances in Electronic Packaging, Vol. 2, ASME 1999, pp 1699-1704.

8. Whalley D. C., Glinsky G., Bailey C. J. and Liu J., "Computational Modelling of the Anisotropic Conductive Adhesive Assembly Process," Proceedings of the 3rd International Symposium on Electronics Materials and Packaging, November 2001, Jeju, Korea, pp393-398.

9. Dou G., Chan Y. C. and Liu J., "Electrical Conductive Characteristics of Anisotropic Conductive Adhesiv Paritcles," Journal of Electronic Packaging, ASME, Vol. 125, December 2003, pp609-616.

10. Holm R., "Electric Contacts, Theory and Application," Springer-Verlag, Berlin, Herdelberg, New york, 1967

11. Oguible C. N., Mannan S. H., Whalley D. C. and Williams D. J., "Conduction Mechnisms in Anisotropic Conducting Adhesive Assembly," IEEE Transctions, Packaging, and Manufacturing Technology, Part A, Vol. 21 No. 2, June 1998, pp235-242.

12. Kristiansen H., Gullisken N., Haugerud H. and Friberg R., "Characterisation of Electrical Contacts Made by Non-conductive Adhesive," 1998, pp345-350

13. Kristiansen H., Bjøneklett A., "Fine Pitch Connection to Rigid Substrates using Non-Conductive Epoxy Adhesvie," Journal of Electronics Manufacturing, Chapman \& Hall, Feburary 1992, pp7-12.

14. Yim M. J, Paik K. W., "Design and Understanding of Anisotropic Conductive Films (ACF's) for LCD Packaging," IEEE Transactions On Components, Packaging, and Manufacturing Technology, part A, Vol. 21, No. 2, June 1998, pp226-234.

15. Johson K: L., "Contact Mechanics," Cambridge, U. K., Cambridge University Press, 1985, pp 93.

16. Chiang K. N., Chang C. W. and Lin J. D., "Analysis of ACA/ACF package Using Equivalent Spring Mehtod," Electronics Pakcaging Technology Conference, 2000, pp110-116.

17. Shi F. G., Abdullah M., Chungpaiboonpatana S., Okuyama K., Davidson C. and Adams J. M., "Electrical Conduction of Anisortropic Conductive
Adhesive: Effect of Size Distribution of Conducting Filler Particles," Matrial Science in Semiconduting Processing 2, 1999, pp263-269.

18. Constable J. H., "Analysis of ACA Contact Resistance," Proceeding of IPACK03, Intrnational Electronic Packaging Technical Conference and Exhibition, July 6-11, 2003, pp1-8.

19. Määttänen J., "Contact Resistance of Metalcoated Polymer Paricles Used in Anisotropically Conductive Adhesices," Soldering \& Surface Mount technology, 15/1, 2003, pp12-15.

20. Wu, S. X., Hu, K. X. and Yeh, C. P., "Contact Reliability modelling and material behavior of conductive adhesvie under thermomechanical loads," conductive Adhesvie for Electrcis Packaging, Chapter 6, Electrochemical Publication Ltd, Isle of Man, p. 121.

21. Liu J, "ACA Bonding Technology for Low Cost Electronics Packaging Applications-Current Status and Remaining Challenges," Soldering \& Surface Mount technology, 13/3, 2001, pp39-57. 